\title{
TRAUMA PENETRANTE EM LOBO FRONTAL COM INTERVENÇÃO SEM SEQUELA EM JOVEM ACIDENTADA DE TRÂNSITO.
}

\section{PENETRANT TRAUMA IN FRONTAL LOBE WITH AN INTERVENTION WITHOUT SEQUELS IN A TRANSIT-ACCIDENTED YOUNG GIRL}

Marcos Paulo da Silva1, Luciano Batista de Paula2; Thiago Francisco Coriolano Aguiar de Souza ${ }^{3}$, Ingrid Almeida Padilha ${ }^{4}$, Fábia Coelho de Araújo ${ }^{5}$, Mônica Mendonça Vieira Marcolino ${ }^{6}$. \begin{abstract}
RESUMO
Introdução: O Traumatismo Crânio-Encefálico é uma das principais causas de morbimortalidade decorrentes de trauma no Brasil e no mundo.

Objetivo: Relatar o caso de uma paciente vítima de trauma penetrante em região frontal após acidente automobilístico e intervenção sem sequelas.

Métodologia: Realizou-se um relato de caso sobre um atendimento de uma paciente do sexo feminino, 19 anos, admitida no Hospital Regional Público de Gurupi após ser vítima de acidente automobilístico. A paciente viajava de ônibus quando o veículo se envolveu em um acidente sendo a paciente arremessada para fora do veículo. Foi conduzida pelo SAMU do local do acidente para o Hospital de Referência devido TCE com fratura exposta e corpo estranho em região frontal à direita.
\end{abstract}

Resultados e Discussão: Paciente previamente hígida evoluiu com rabaixamento do nível de consciência sendo submetida a procedimento cirúrgico de emergência para remoção de corpo estranho e drenagem de hematoma. $O$ procedimento foi realizado sem intercorrências, entretanto 0 pós-operatório imediato foi de alta complexidade, devido a paciente evoluir com choque séptico, permanecendo 5 dias na UTI com suporte hidroeletrolítico rigoroso. Após 12 dias de pós-operatório paciente recebeu alta médica ao apresentar melhora expressiva.

Considerações Finais: $O$ traumatismo crânioencefálico é o principal fator determinante da mortalidade e da morbidade decorrentes do trauma. A predição do prognóstico é um dos principais problemas associados ao traumatismo crânioencefálico grave. Evidenciando-se o caso de uma paciente jovem, 19 anos, que por gozar de um atendimento rápido, obteve prognóstico favorável com êxito/sucesso.

Descritores: Psoríase. Raios Solares. Tratamento.

\section{INTRODUÇAO}

O traumatismo crânio-encefálico (TCE) é uma importante causa de mortalidade e morbidade em países ocidentais. Dentre das principais causas de TCE pode-se destacar acidentes com veículos em

\section{ABSTRACT}

Introduction: Cranio-Encephalic Trauma is one of the main causes of morbidity and mortality due to trauma in Brazil and in the world.

Objective: To report the case of a patient victim of penetrating trauma in frontal region after auto accident and intervention without sequelae.

Methodology: A case report on a care of a female patient, 19 years old, admitted to the Regional Public Hospital of Gurupi after being a victim of an auto accident was carried out. The patient traveled by bus when the vehicle was involved in an accident and the patient was thrown out of the vehicle. It was conducted by the SAMU from the accident site to the Reference Hospital due TBI with exposed fracture and foreign body in the right frontal region.

Results and discussion: Patient previously healthy evolved with lowering of the level of consciousness being submitted to emergency surgical procedure for foreign body removal and hematoma drainage. The procedure was performed without intercurrences. However, the immediate postoperative period was highly complex, due to the patient developing septic shock, remaining 5 days in the ICU with rigorous hydroelectrolytic support. After 12 postoperative days, the patient was discharged when he presented significant improvement.

Final considerations: Cranio-encephalic trauma is the main determinant of mortality and morbidity resulting from trauma. Prediction of prognosis is one of the main problems associated with severe head trauma. It was evidenced the case of a young patient, 19 years old, who, due to the rapid attendance, obtained favorable prognosis with success.

Descriptors: Psoriasis. Sun rays. Treatment.
'Acadêmico do $11^{\circ}$ período do curso de medicina do Centro Universitário UnirG. Gurupi-TO, Brasil. Email:

marcosmps13@gmail.com

ENDEREÇO PARA CORRESPONDÊNCIA:

Av. Bernanrdo Sayão, n.

1099, Miranorte, Tocantins,

Brasil, CEP 77660-000.

Telefone: (63) 98427-3543. 
10.18606/2318-1419/amazonia.sci.health.v6n1p22-25 Revista Amazônia Science \& Health.

2018 Jan/Mar
Silva MP, De Paula LB, Souza TFCA, Padilha IA, Araújo FC, Marcolino MMV.

Penetrant trauma in frontal lobe with an intervention without sequels in a transit-accidented young girl. segurança rodoviária e ocupacional, estes mantêmse como a principal causa de mortalidade e morbilidade entre os adultos jovens. ${ }^{2,3,4,5}$

É difícil apurar a incidência real dos TCE por várias razões: sub-diagnóstico, ausência de procura de cuidados médicos em situações de TCE ligeiro e falta de sistemas de monitorização e registro em muitas unidades de saúde ${ }^{2,6,7,8}$.

Dentre a faixa etária acometida por traumas automobilísticos, destaca-se o grupo dos adolescentes e adultos jovens. Os traumas ocorrem preferencialmente, em homem (81,9\%) na fase adulto jovem (34,4\%), sendo a maioria decorrente automobilístico $(46,2 \%)$ e violência interpessoal $(22,5 \%)^{9}$.

Nos Estados Unidos da América (EUA) em 2003 teriam ocorrido 1.565.000 TCE resultando em 1.224.000 admissões em Serviços de Urgência, 290.000 internamentos e 51.000 mortes, o que corresponde a mais de 60 mil milhões de dólares de despesa anual (não estando incluídas neste valor as consequências económicas para as famílias ou cuidadores) ${ }^{10}$.

Estudos mais recentes demonstram uma incidência de TCE de aproximadamente 103/100000 habitantes nos EUA. Apesar de a incidência ser ainda muito elevada, tem vindo a observar-se uma diminuição da mortalidade associada ao TCE nas sociedades desenvolvidas, resultado da melhoria da prevenção, dos sistemas de emergência médica e da implementação de guidelines para avaliação e tratamento do traumatizado crânio-encefálico ${ }^{2,7,11}$.

\section{METODOLOGIA}

O estudo trata-se de relato de experiência de intervenção cirúrgica emergencial de trauma craniano realizada em um hospital público na região sul do Tocantins. Assim, para que se pudesse realizar o mesmo, bem como divulgar as imagens das etapas da evolução do caso, foi solicitada autorização por escrito de familiar da paciente informando os riscos e os benefícios.

Paciente A.S.A., feminino, 19 anos, foi admitida no Hospital Regional Público de Gurupi após envolver em acidente na BR 153 no trecho entre as cidades de Alvorada-TO e Figueirópolis-TO no dia 31 de maio de 2014. A paciente estava em um ônibus viajando para Brasília quando o veículo se envolveu em um acidente e a mesma foi ejetada para fora do veículo.

A mesma foi atendida e conduzida pelo SAMU (Serviço de Atendimento Móvel de Urgência) do local do acidente para o Hospital de Referência devido TCE com fratura exposta e corpo estranho em região frontal à direita (Figura 1).

Figura 1 - Paciente no momento da admissão, com presença de corpo estranho perfurando o crânio.

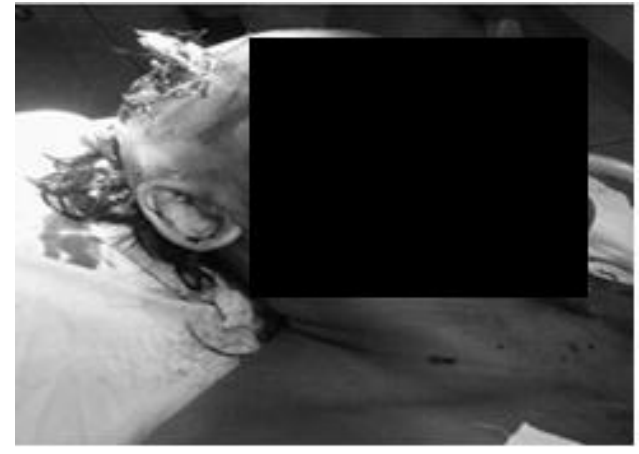

Paciente previamente hígida negava etilismo, tabagismo e doenças prévias. Ao exame apresentava-se confuso, regular estado geral, palidez de mucosas, extremidades frias, evoluindo com hemiplegia à $\mathrm{E}$. Devido rebaixamento do nível de consciência foi submetida a procedimento cirúrgico de emergência para remoção de corpo estranho e drenagem de hematoma, não seria possível realizar exame de imagem devido instabilidade hemodinâmica.

\section{Descrição do procedimento cirúrgico}

\section{Figura 2 - Osso parietal direito com perfuração devido o corpo estranho.}

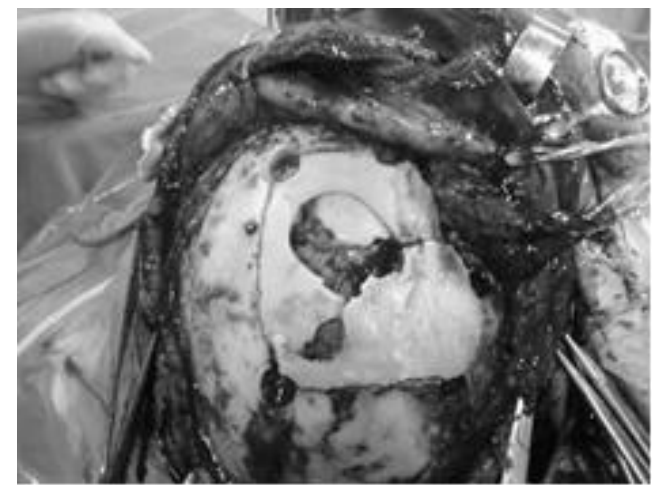

Paciente em decúbito dorsal, sob anestesia geral, com cabeça apoiada em coxim. Antissepsia, assepsia e colocação dos campos. Incisão de Becker à direita, hemostasia por planos, craniotomia em 5 furos em volta do corpo estranho com evidencia de fratura estendendo até a região temporal baixa. Retirada da peça óssea, abertura da dura-máter e retirada do corpo estranho (pedaço de madeira) com mais ou menos $6 \mathrm{~cm}$ em região frontal média. Lavagem exaustiva da cavidade; Drenagem do hematoma extradural; Retirada de duas espículas ósseas, cabelo e madeira. Hemostasia; Revisão do sitio cirúrgico; Fechamento da dura-máter com enxerto de fáscia; Volta de partes do osso e fixação do mesmo. Fechamento por planos; Dreno extradural; Sutura da pele (figura 3). 
10.18606/2318-1419/amazonia.sci.health.v6n1p22-25 Revista Amazônia Science \& Health.
Silva MP, De Paula LB, Souza TFCA, Padilha IA, Araújo FC, Marcolino MMV.

Penetrant trauma in frontal lobe with an intervention without sequels in a transit-accidented young girl.
Figura 3 - Corpo estranho retirado.

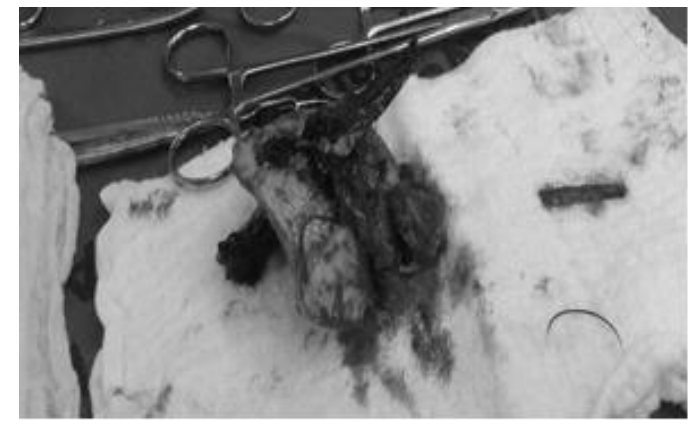

Exames complementares

A) Hemoglobina e hematócrito: 10,5 - 31,3; Leucócitos: 17.300; Bastonetes: 6\%; Segmentados $75 \%$; Eosinófilos $1 \%$.

B) Tomografia de crânio: Edema em sítio cirúrgico, hematoma epidural, fragmentos em região de lobo frontotemporal à direita e desvio da linha média.

\section{RESULTADOS E DISCUSSĀO}

Procedimento cirúrgico foi realizado sem intercorrências. O pós-operatório (PO) imediato foi de difícil controle, pois a paciente evoluiu com choque séptico, sendo submetida a rigoroso suporte hidroeletrolítico em UTI onde permaneceu por 5 dias.

Durante internação em UTI permaneceu sedada, intubada e confortável em VM (Ventilação mecânica). Pupilas isofotoreagentes, hemoestável e com diurese presente. No $1^{\circ} \mathrm{PO}$ foi realizada TC de crânio que apresentou sangramento, hematoma epidural discreto e fragmentos em lobo frontotemporal à direita. No $3^{\circ} \mathrm{PO}$ foi retirado o dreno, cicatriz cirúrgica seca, sem sinais flogísticos e foi programada a extubação devido boa evolução. No $4^{\circ} \mathrm{PO}$ a paciente apresentava-se acordada, atendia ao comando verbal e melhorou déficit à esquerda. Foi iniciado anticonvulsivante.

No $5^{\circ} \mathrm{PO}$ a ferida operatória estava seca. Paciente sem febre e com melhora neurológica significativa. $6^{\circ} \mathrm{PO}$ Paciente consciente teve pico febril. Negou cefaléia e vômito. Apresentou melhora de movimento em membro inferior esquerdo, queixou de dor e ainda apresentava plegia de membro superior esquerdo. Alimentando por via oral e sem sonda vesical. No $7^{\circ}$ e $8^{\circ} \mathrm{PO}$ manteve déficit em MSE, porém manteve-se afebril.

No $9^{\circ} \mathrm{PO}$ a paciente referiu cefaléia holocraniana tipo pontada, porém apresentou melhora de déficit motor de membro superior esquerdo. Verbalizando e deambulando. No $10^{\circ} \mathrm{PO}$ foi retirado os pontos de ferida operatória e realizada nova TC de crânio. $11^{\circ} \mathrm{PO}$ a paciente apresentou melhora clínica significativa. Em TC de crânio edema em sitio cirúrgico, reabsorção de hematoma extradural e ainda permanecia desvio da linha média.

Paciente teve alta no $12^{\circ}$ PO lúcida, orientada, sem queixas, com melhora de paresia de MSE, sem febre, vômito ou cefaleia, deambulando.

Apesar das medidas preventivas presentes nas estradas e rodovias do país, os condutores exercem bastante influência em termos de responsabilidade de condução, possuindo papel importante no desenvolvimento de TCE decorrente de má conduta no trânsito ${ }^{3}$.

A paciente do presente caso clínico se enquadrava dentro dos achados epidemiológicos mais comuns naqueles que sofrem um trauma crânio-encefálico (TCE): trauma por acidentes automobilísticos envolvendo adultos jovens ${ }^{9}$.

A lesão encefálica definitiva estabelecida no TCE resulta de vários mecanismos fisiológicos e patológicos que ocorrem desde o momento do acidente até dias ou semanas e é dividida em lesão primária e secundária. A lesão primária ocorre no momento do trauma e é causada por várias etiologias: armas de fogo e armas brancas, por exemplo, enquanto que a lesão secundária consiste em agressões, tanto intra quanto extracerebrais, no parênquima que começam após o momento do acidente, ocasionando a morte das células locais ${ }^{12}$,

Sendo assim, é necessária uma intervenção precoce e efetiva a fim de evitar possíveis sequelas, que são variáveis de acordo com o local acometido e o grau de acometimento ${ }^{14,15}$.

Para realizar o tratamento de um paciente com TCE deve-se ter em mente a causa do trauma, o nível de acometimento das estruturas envolvidas por meio da realização de exames de neuroimagem e, além disso, fazer o seguimento do paciente com exames de avaliação neurológica ${ }^{16}$.

No caso apresentado, a evolução póscirúrgica inicial demonstrou-se favorável, apesar do pós-operatório imediato de difícil controle. A intervenção cirúrgica foi realizada a fim de retirar o corpo estranho e de minimizar as possíveis sequelas do trauma.

\section{CONSIDERAÇOESS FINAIS}

O traumatismo crânio-encefálico é o principal fator determinante da mortalidade e da morbidade decorrentes do trauma. A predição do prognóstico é um dos principais problemas associados ao traumatismo crânio-encefálico grave, já que o valor preditivo variável da avaliação clínica complica a identificação de pacientes com maior risco para desenvolvimento de lesões secundárias e desfecho fatal.

A tomografia computadorizada (TC) é o exame de imagem de escolha no manejo da vítima de TCE na sala de emergência. Através desse 
10.18606/2318-1419/amazonia.sci.health.v6n1p22-25 Revista Amazônia Science \& Health.

2018 Jan/Mar
Silva MP, De Paula LB, Souza TFCA, Padilha IA, Araújo FC, Marcolino MMV.

Penetrant trauma in frontal lobe with an intervention without sequels in a transit-accidented young girl. exame, hematomas podem ser rapidamente diagnosticados, favorecendo, quando indicado, tratamento cirúrgico precoce. A TC tem, no entanto, baixa sensibilidade no diagnóstico de lesões não hemorrágicas, o que explica a pobre correlação, muitas vezes observada, entre os achados tomográficos e a pontuação na Escala de Coma de Glasgow. A TC deve ser repetida se houver deterioração do quadro clínico, uma vez que lesões já diagnosticadas podem aumentar ou novas lesões podem se desenvolver, principalmente nas primeiras 12 a 24 horas após o trauma. E assim evidencia-se o caso de uma paciente jovem, 19 anos, que por gozar de um atendimento rápido, obteve prognóstico favorável com uma ótima recuperação, demonstrando assim que pacientes jovens possuem uma melhor recuperação, decorrente de acidentes graves, quando atendidos precocemente.

\section{REFERENCIAS}

1. Carlotti CGJ, Boullosa, JLR, Dias LAA, Oliveira RS, Colli BO. Traumatismos craniencefálicos. Medicina (Ribeiräo Preto).1995;28(4):765-76.

2. Winn HR, Bullock M, Hovda D, Schouten J, Maas A, Shahlaie K, Zwienenberg-lee M, Muizelaar J et al. Youmans Neurological Surgery: Epidemiology of Traumatic Brain Injury". 2011; 323,327,331(4):3270-3275;3362-3379.

3. Koizumi MS et al. Morbimortalidade por traumatismo crânio-encefálico no município de São Paulo, 1997. Arquivos de Neuro-Psiquiatria. 2000;58(1):81-89.

4. Greenberg RP, Stablein DM; Becker DP. Noninvasive localization of brain-stem lesions in the cat with multimodality evoked potentials: correlation with human head-injury data. Journal of Neurosurgery. 1981;54(6):740-750.

5. Maas Al, Hukkelhoven CW, Marshall LF, Steyerberg EW. Prediction of outcome in traumatic brain injury with computed tomographic characteristics: a comparison between the computed tomographic classification and combinations of computed tomographic predictors. Journal of Neurosurgery. 2005; 57(6):1173-1182.

6. Martins CBG, Andrade SM. Causas externas entre menores de 15 anos em cidade do Sul do Brasil: atendimentos em pronto-socorro, internações e óbitos. Rev. Brasileira de Epidemiologia. 2005; 8(2):194-204.

7. Meixensberger J, Kunze E, Barsay E, Vaeth A, Roosen K. Clinical cerebral microdialysis: brain metabolism and brain tissue oxygenation after acute brain injury. Neurological research. 2001; 23:801-6.

8. Cochan A, Scaife ER, Hansen KW, Downey EC. Hyperglycemia and outcomes from pediatric traumatic brain injury. The Journal of Trauma. 2002; 55:1035-8.

9. Vieira CL, Araújo DCDC, Ribeiro MLS, Laureano Filho JR. Lesão de tecido mole em pacientes vítimas de trauma buco-maxilo-facial. Revista de Cirurgia e Traumatologia Buco-maxilo-facial. 2013; 13(1):97-104.

10.Oliveira E et al. Traumatismo Crânio-Encefálico: Abordagem Integrada. Acta Médica Portuguesa. 2012; 25(3).

11. Oliveira E, Lavrador JP, Santos MM, Antunes JL. Traumatismo crâncio-encefálico: abordagem integrada. Acta Médica Portuguesa. 2012;25(3):179-192.

12.Andrade AF, Paiva WS, Amorim ROL, Figueiredo EG, Neto ER, Teixeira MJ. Mecanismos de lesão cerebral no traumatismo cranioencefálico. Revista da Associação Médica Brasileira. 2009;55(1):75-81.

13. Maset A, Andrade A, Martucci $S$ et al. Epidemiologic features of head injury in Brazil. Arq. Bras. Neurocir. 1993;12(4):293-302.

14.Reilly P, Bullock R. Head injury: Pathophysiology and Management. $2^{\mathrm{a}}$ ed. London: CRC Press; 2005.

15. Teasdale G, Jennett B. Assessment of coma and impaired consciousness: a practical scale. The Lancet. 1974;2(7872):81-84.

16.Chaves MLF, Finkelsztejn A, Stefani, MA. Rotinas em Neurologia e Neurocirurgia. Porto Alegre: Artmed; 2008 